\title{
Cis Regulatory Elements in Regulation of Plant Gene Expression: An Overview
}

\section{Rajesh Mehrotra, Chetna Sangwan, Zaiba Hasan Khan and Sandhya Mehrotra}

Department of Biological Sciences, Birla Institute of Technology and Science, Pilani, Rajasthan 333031, India

\section{Short Commentary}

Gene expression is an extensively controlled process that occurs at various levels, transcription being one of the most crucial. It depends on a variety of interactions mediated by the core promoter region, sequence specific DNA binding proteins and their cognate promoter elements. Promoters comprise cis regulatory elements, clusters of short sequences that provide binding sites for transcription factors. According to PLACE database, there are 469 cis regulatory elements present in plants. The ACGT core sequence has been established as a functionally important cis element in several promoters that respond to different stimuli like light, anaerobiosis, jasmonic acid and hormones such as salicylic acid, abscisic acid and auxin. It frequently regulates gene expression in synergy with other cis elements (DRE, CRE, LRE, etc). Promoter activity is largely affected by the copy number, inter motif distance, position, deletions or mutations in the core sequences of the cis regulatory regions. Our research is aimed at understanding the mechanistics of transcriptional regulation, focusing on the role of cis regulatory modules in regulation of gene expression.

We did comparative genomic analysis of four different plant genomes. Results indicated that certain spacer lengths are preferred over others that suggests that these lengths might be involved in vital physiological processes. Using gene expression databases we also observed trends suggesting that co occuring ACGT elements are responsible for gene regulation in response to exogenous stress. Conservation in patterns of ACGT (N) ACGT among orthologous genes also indicated the possibility that emergence of functional significance across species was a result of parallel evolution of these cis elements [1].

Another study from our group in Arabidopsis thaliana indicated that two copies of ACGT elements separated by 5 nucleotides gave the highest activation of the reporter gene which imparted salicylic acid inducibility. However, a spacer length of 25 nucleotides allowed the promoter to be induced by abscisic acid but not salicylic acid. The differential induction is expected to involve the recruitment of different bZIP transcription factors. This suggests that a change in spacing between two copies of a given motif can alter the signal pathway to which a promoter responds [2].

In silico analysis also showed that ACGT elements separated by 25 nucleotides are more frequent than those by 10 base pairs suggesting directed evolution of larger motifs in Arabidopsis thaliana genome. This shows that under some evolutionary forces this interval was favored since this distance may cause changes in the level of gene expression or in its robustness against variation in transcription factor concentration [3].

Genome wide analysis of Arabidopsis thaliana revealed an exceptionally high frequency of AAAGN7CTTT motif, AAAG and CTTT being the binding sites for dof proteins. However, when the orientation was changed to CTTTN7AAAG this tendency was not observed. This is likely that this sequence combination might have a functional significance [4].

Our work in the protein phosphatase 2C (PP2C) like promoter of Arabidopsis thaliana revealed that AACA element negatively regulated the expression of the above promoter, even in the presence of inducers [5].

We have been also working on designing of promoter that can be constructed by placing one or more different cis regulatory elements individually or in combination. Exploration of natural promoter architecture and the concept of cis engineering have enabled fine tuning of single or multiple transgene expression in response to variations in the chemical, physiological and environmental stimuli and in the form of bidirectional promoters it also provided a unique answer to various problems in crop improvement [6].

We believe that our research will be an inspiration for designing promoters where specific interactions could be directed.

\section{References}

1. Mehrotra R, Sethi S, Zutshi I, Bhalothia P, Mehrotra S, et al. (2013) Patterns and evolution of ACGT repeat cis-element landscape across four plant genomes BMC Genomics 14: 203.

2. Mehrotra R, Mehrotra S (2010) Promoter Activation by ACGT in Response to Salicylic and Abscisic Acids Is Differentially Regulated by the Spacing between Two Copies of the Motif. Journal of Plant Physiology 167: 1214-1218.

3. Mehrotra R, Yadav A, Bhalothia P, Karan R, Mehrotra S, et al. (2012) Evidence for Directed Evolution of Larger Size Motif in Arabidopsis thaliana Genome. The Scientific World Journal. Article ID: 983528.

4. Mehrotra R, Jain V, Shekhar C, Mehrotra S (2014) Genome wide analysis of Arabidopsis thaliana reveals high frequency of AAAGN7CTTT motif Published by Elsevier B.V, Meta Gene 2: 606-615.

5. Bhalothia P, Alok A, Mehrotra S, Mehrotra R (2013) AACA Element Negatively Regulates Expression of Protein Phosphatase 2C (PP2C) like Promoter in Arabidopsis thaliana.American Journal of Plant Sciences 4: 549-554.

6. Mehrotra R, Gupta G, Sethi R, Bhalothia P, Kumar N, et al. (2011) Designer promoter: an artwork of cis engineering, Plant Molecular Biology 75: 527-536.
*Corresponding author: Rajesh Mehrotra, Department of Biological Sciences Birla Institute of Technology and Science, Pilani, Rajasthan 333031, India, Tel: +91-1596-51-5666; E-mail: rajmeh25@hotmail.com

Received September 09, 2015; Accepted October 17, 2015; Published October 19, 2015

Citation: Mehrotra R, Sangwan C, Khan ZH, Mehrotra S (2015) Cis Regulatory Elements in Regulation of Plant Gene Expression: An Overview. Gene Technol 4: 129. doi: 10.4172/2329-6682.1000129

Copyright: (c) 2015 Mehrotra R, et al. This is an open-access article distributed under the terms of the Creative Commons Attribution License, which permits unrestricted use, distribution, and reproduction in any medium, provided the original author and source are credited. 